Published in final edited form as:

J Aging Health. 2019 September ; 31(8): 1339-1352. doi:10.1177/0898264318778417.

\title{
Cardiovascular Health Is Associated With Disability Among Older Community Dwelling Men and Women
}

\author{
Yichen Jin, MSPH $^{1}$, Toshiko Tanaka, $\mathrm{PhD}^{2}$, Yan Ma, $\mathrm{PhD}^{1}$, Stefania Bandinelli, $\mathrm{MD}^{3}$, Luigi \\ Ferrucci, MD, $\mathrm{PhD}^{2}$, Sameera A. Talegawkar, $\mathrm{PhD}^{1}$ \\ ${ }^{1}$ The George Washington University, DC, USA \\ ${ }^{2}$ National Institute on Aging, Baltimore, MD, USA \\ ${ }^{3}$ Azienda Sanitaria di Firenze, Florence, Italy
}

\section{Abstract}

Objective: The aim of this study was to investigate whether an index of overall cardiovascular health $(\mathrm{CVH})$ is associated with disability in older individuals.

Method: Data on 925 participants of the InCHIANTI study (Invecchiare in Chianti, aging in the Chianti area, 265 years, 55\% women) with median follow-up of 9 years were used. CVH score was assessed by smoking status, physical activity, body mass index, diet quality, blood pressure, plasma cholesterol, and fasting blood glucose. Disability was examined using instrumental activities of daily living (IADL disabilities $>0$ vs. 0 ) and activities of daily living (ADL disabilities $>0$ vs. 0 ). Generalized estimating equations and Cox models assessed relationships between baseline $\mathrm{CVH}$ with disability and worsening over 9 years.

Results: A 1-point increase in the CVH score was associated with $23 \%$ and $17 \%$ of lower odds of ADL $(p<.001)$ and IADL $(p<.001)$ disability and was protective of worsening of disability over 9 years. Cox models demonstrated that a 1-point increase in CVH score was associated with lower hazards of both ADL (hazard ratio $[\mathrm{HR}]=0.86, p=.005)$ and IADL (HR $=0.91, p=.007)$ disability.

Discussion: Among older individuals, better $\mathrm{CVH}$ was associated with lower risk of disability and worsening over 9 years.

\section{Keywords}

InCHIANTI study of aging; ADL; IADL; worsening disability

\footnotetext{
Corresponding Author: Sameera A. Talegawkar, Department of Exercise and Nutrition Sciences, Milken Institute School of Public Health, The George Washington University, 950 New Hampshire Avenue NW, 2nd Floor, Washington, DC 20052, USA. sameera.talegawkar@alumni.tufts.edu.

Declaration of Conflicting Interests

The authors declared no potential conflicts of interest with respect to the research, authorship, and/or publication of this article.
} 


\section{Introduction}

It is projected that by 2030, the worldwide population of individuals 65 years and older will have increased from 550 to 973 million (U.S. Census Bureau, 2015; Vincent \& Velkoff, 2010). As life expectancy increases, the quality of life is a significant public health concern with disability in late-life becoming a pressing public health issue. Population aging is currently at the center of public policy debate because of its potential for significantly impacting health care expenditures of countries. Economists believe that while aging is an important contributor to these expenses, several factors either directly impact or interact with population aging to impact these (United Nations Department of Economic and Social Affairs, Population Division, 2015). One such factor is disability, more commonly associated with chronic diseases such as cardiovascular disease (CVD), cancer, and dementia, which is more prevalent in older individuals (Millán-Calenti et al., 2010), resulting in increased health care costs associated with hospitalization or long-term care (Crimmins, 2004). Therefore, investigation of the factors associated with disability among elderly is important for formulating public health strategies to improve physical function and maintain physical well-being in late-life.

Cardiovascular health $(\mathrm{CVH})$ is a concept that was proposed and defined by the American Heart Association (AHA) in 2010 to identify factors that together were associated with a lower risk of CVD and stroke, and the Life's Simple 7 (LS7), a metric that includes seven health behaviors and factors, including smoking status, physical activity, body mass index (BMI), diet quality, blood pressure, total cholesterol, and fasting plasma glucose, has been used to assess CVH (Lloyd-Jones et al., 2010). Studies have shown that higher overall scores for this metric and its components, indicating better $\mathrm{CVH}$, are associated with a lower risk of chronic disease, age-related declines in physical and cognitive functioning, and mortality (Folsom et al., 2011; Jin, Tanaka, Bandinelli, Ferrucci, \& Talegawkar, 2016; Jin et al., 2017; Rebholz et al., 2016; Talegawkar, Jin, Kandula, \& Kanaya, 2017; Thacker et al., 2014). Two cross-sectional studies have examined associations between CVH and disability. Using nationally representative data from non-institutionalized individuals 20 years and older from the National Health and Nutrition Examination Survey (NHANES) from 2005 to 2012, a 1point increase in the number of $\mathrm{CVH}$ metrics within an ideal level was associated with a $16 \%$ reduction in the odds of disability (Devulapalli, Shoirah, \& Dhamoon, 2016), and using data from the Chilean National Health Survey 2009-2010, 65 years and older individuals with five to seven ideal metrics had $49 \%$ lower odds of disability compared with those with zero to two ideal metrics (García-Hermoso, Ramírez-Vélez, Ramirez-Campillo, \& Izquierdo, 2017). In addition, in a longitudinal cohort of middle aged and older men and women belonging to the Northern Manhattan Study, after a follow-up of 13 years, participants with at least five of the metrics in the ideal range had $45 \%$ lower odds of disability compared with those with less than two of the metrics (Dhamoon, Dong, Elkind, \& Sacco, 2015). While these investigations have examined the associations between CVH and disability, there are no studies that we are aware of examining the longitudinal role of $\mathrm{CVH}$ assessed using the LS7 metric and progression of disability among older individuals.

Therefore, in these analyses, we examined the associations between $\mathrm{CVH}$, assessed at baseline, and the odds for and worsening of disability assessed using activities of daily living 
(ADL) and instrumental activities of daily living (IADL) in a cohort of community dwelling men and women 65 years and older over a 9-year follow-up. We hypothesized that a higher score, indicating better $\mathrm{CVH}$, would be associated with lower odds of disability and its worsening.

\section{Method}

We used data from the InCHIANTI cohort, a longitudinal study of mostly older individuals randomly selected using a two-stage stratified sampling procedure from two cities, Greve in Chianti and Bagno a Ripoli, in Tuscany, Italy, in 1998-2000 with 1,453 participants recruited. The detailed description of the InCHIANTI study procedures has been provided elsewhere (Ferrucci et al., 2000). All respondents signed informed consent, and the Italian National Institute of Research and Care on Aging Ethical Committee approved the study protocol.

For these analyses, we excluded participants less than 65 years of age $(n=287)$, those diagnosed with dementia $(n=82)$, those with implausible daily energy intakes (defined as below $600 \mathrm{kcal} / \mathrm{d}$ or above $4,000 \mathrm{kcal} / \mathrm{d}, n=14)$, those with a BMI of less than $18.5 \mathrm{~kg} / \mathrm{m}^{2}$ $(n=4)$, and those missing components of $\mathrm{CVH}(n=141)$. The final analytical sample included 925 participants at baseline, and 788, 715, and 597 participants with available ADL and IADL at 3-year (2001-2003), 6-year (2004-2006), and 9-year (2007-2009) follow-ups, respectively.

\section{ADL and IADL Disability}

In the InCHIANTI cohort, disability status was assessed as part of cohort procedures at baseline and each follow-up visit, and was operationalized using assessments of ADL and IADL (Katz, Ford, Moskowitz, Jackson, \& Jaffe, 1963; Lawton \& Brody, 1969). The ADL questionnaire includes six functional tasks: bathing, dressing, toileting, getting into and out of bed, continence, and eating. While the IADL includes eight daily tasks: using the telephone, shopping, food preparation, light housework, laundering, transportation, taking medications, and handling finances. The number of ADL and IADL disabilities was defined as the number of these daily tasks that participants were unable to accomplish, and the number of ADL and IADL disabilities greater than 0 was considered as having disability.

Worsening of ADL and IADL disability at each follow-up was defined as increase in the number of ADL and IADL disability at follow-up from baseline and treated as dichotomous outcome compared with no change or decrease in the number of reported ADL and IADL disability.

\section{Overall CVH Score}

$\mathrm{CVH}$ was assessed by seven health behaviors and factors including smoking status, physical activity, BMI, diet quality, blood pressure, total cholesterol, and fasting plasma glucose, and a score of 0 (poor), 1 (intermediate), or 2 (ideal) was assigned to participants for each component based on their status according to AHA LS7 criteria (Lloyd-Jones et al., 2010). This results in a score ranging from 0 to 14 , with 14 having perfect CVH. 
We modified the criteria for smoking status and physical activity based on data available for the cohort. According to the LS7 criteria, smoking status is classified as never or quit $>12$ months, former (quit $\leq 12$ months), and current smoker, and physical activity is defined as none, 1 to $149 \mathrm{~min} /$ week moderate, or 1 to $74 \mathrm{~min} /$ week vigorous, or 1 to $149 \mathrm{~min} /$ week moderate + vigorous activity, and $\geq 150 \mathrm{~min} /$ week moderate, or $\geq 75 \mathrm{~min} /$ week vigorous or $250 \mathrm{~min} /$ week moderate + vigorous activity. In this study, smoking status was classified as never, former, and current smoking within 3 years. For physical activity, participants who were inactive or having some walking were classified as inactive; those who had light exercise for 2 to $4 \mathrm{hr} /$ week were classified as light active; and those with light exercise for more than $4 \mathrm{hr} /$ week, moderate exercise for at least 1 to $2 \mathrm{hr} / \mathrm{week}$, or intense exercise many times/week were classified as active. The data for smoking status and physical activity were collected at the home interview using standard questionnaires.

A validated food frequency questionnaire (FFQ) previously developed for the Italy site of European Prospective Investigation on Cancer and Nutrition investigations was used to assess self-reported dietary intakes (Bartali et al., 2004; Pisani et al., 1997). We operationalized diet quality by examining adherence to a Mediterranean-style diet that is more consistent with the dietary pattern in the geographical region where participants resided. The Mediterranean diet score (ranged from 0 to 9 ) was calculated by assessing the intakes of nine food groups and nutrients, and 1 point was assigned for each beneficial dietary item including vegetables, legumes, fruits and nuts, cereal, fish, monounsaturated-tosaturated fatty acid ratio if intake was greater than the sex-specific median, and for meat and dairy intakes if less than sex-specific median (considered detrimental), and moderate intake of alcohol (Trichopoulou, Costacou, Bamia, \& Trichopoulos, 2003).

Weight (in $\mathrm{kg}$ ) and height (in $\mathrm{m}$ ) and blood pressure were measured at study clinic. BMI (in $\mathrm{kg} / \mathrm{m}^{2}$ ) was calculated as weight divided by the square of height. Blood pressure was measured thrice, 2 min apart in supine position using standard mercury sphygmomanometer. The last two measures were conducted on the arm with the higher systolic blood pressure at the first measure, and the average value of the last two measures was used for analyses. Information on medication use was collected through home interview (Ferrucci et al., 2000).

Total cholesterol and plasma glucose were measured through blood tests. Blood samples were collected after participants had fasted at least $8 \mathrm{hr}$. Total cholesterol was measured using commercial enzymatic tests (Roche Diagnostics), and fasting plasma glucose was measured by an enzymatic colorimetric assay using a modified glucose oxidase-peroxidase method and a Roche-Hitachi 917 analyzer (Roche Diagnostics, GmbH, Mannheim, Germany).

The detailed information on scoring criteria was summarized in Supplementary Table 1. The overall $\mathrm{CVH}$ score was calculated by summing individual score of each $\mathrm{CVH}$ component. In this cohort, the overall $\mathrm{CVH}$ score ranged from 0 to 12 because no one received ideal level score for all components. We have previously established the associations between this $\mathrm{CVH}$ score and mortality and physical function which showed consistent results with other literatures (Jin et al., 2016; Jin et al., 2017). 


\section{Covariates}

Covariates were selected based on univariate analyses and previously published literature on disability, and included age (years), sex, education (years, only for IADL), presence of chronic diseases (number), impaired cognition, and depression. Chronic diseases, including cancer, heart failure, coronary heart disease, stroke, chronic lung disease, hip arthritis, liver disease, gastrointestinal disease, peripheral arterial disease, Parkinson's disease, and renal disease, were assessed based on the criteria used in the Women's Health and Aging Study (Guralnik, Fried, Simonsick, Kasper, \& Lafferty, 1995). Mini-Mental State Examination (MMSE) was used to assess cognitive status, and a score less than 24 was defined as having impaired cognition (Dick et al., 1984). Depression was identified as having score of at least 16 on the Center for Epidemiological Studies-Depression (CES-D) scale (Milaneschi et al., 2011).

\section{Statistical Analysis}

The baseline CVH score was categorized into tertiles with scores ranging from 0 to 6,7 to 8 , and 9 to 12 for first, second, and third tertile, respectively. Baseline sociodemographic characteristics were reported as mean \pm standard deviation, percentage, or median (interquartile range). ANCOVA, chi-square, or Kruskal-Wallis tests were used to examine differences of baseline sociodemographic characteristics across $\mathrm{CVH}$ score tertiles. $\mathrm{CVH}$ score was analyzed on both continuous scale and tertiles. We used generalized estimating equations (GEE) models with unstructured correlation to assess the relationship between baseline CVH with ADL and IADL disability over a 9-year follow-up, after adjusting for baseline covariates. We also examined the associations between worsening of ADL and IADL disability with CVH score using GEE models. Cox proportional hazards regression models were used to estimate associations between baseline $\mathrm{CVH}$ with time to incidence of ADL and IADL disability in participants without reported disability at baseline after testing for the assumption of proportionality of the hazard ratios. The percent change of the number of ADL and IADL disability, adjusting for baseline status, at each follow-up from baseline was also calculated and assessed across $\mathrm{CVH}$ score tertiles. All covariates and time variable were examined for effect modification; however, these were not significant. Due to the effect of stroke, heart failure, and cancer on disability status, we conducted sensitivity analysis on the association between CVH with ADL and IADL disability with GEE models after excluding participants who reported these at baseline. Also, another sensitivity analysis was conducted to assess associations between $\mathrm{CVH}$ and disability and its worsening after excluding participants with disability at baseline as individuals with disability have less physical activity which could lead to reverse causation. In supplementary analyses, CVH components were classified as health behaviors (smoking status, physical activity, BMI, and diet quality) and health factors (blood pressure, total cholesterol, and plasma fasting glucose), and the longitudinal associations between scores of health behaviors (ranged from 0 to 8 ) and health factors (ranged from 0 to 6 ) with ADL and IADL disability worsening were also examined using GEE models. Stata version 13 (StataCorp., 2013) was used to perform all the analyses, and a $p$ value $<.05$ considered to be statistically significant. 


\section{Results}

Socio-demographic and health characteristics of the cohort at baseline are shown in Table 1. About 55\% of participants were women with a mean age of 74 years. Participants with higher CVH scores had lower number of chronic diseases $(p<.001)$, and did not report ADL $(p=.016)$ and IADL $(p<.001)$ disabilities. Four percent of participants had at least one ADL disability at baseline, while $19 \%$ reported at least one IADL disability.

Adjusted longitudinal associations between $\mathrm{CVH}$ scores with ADL and IADL disability over a 9-year follow-up are presented in Table 2. Among all participants, for ADL, a 1-point increase in the $\mathrm{CVH}$ score was associated with $23 \%$ lower odds of ADL disability ( $p<$. $001)$; similarly, compared with those in lowest tertile of $\mathrm{CVH}$, those in the second and third tertiles of CVH score had $40 \%(p=.019)$ and $67 \%(p<.001)$ lower odds of ADL disability over a 9-year period, respectively. Similar protective associations were observed between CVH and IADL disability among all participants. In sensitivity analysis, after excluding participants with disability at baseline, higher $\mathrm{CVH}$ scores were still significantly associated with lower odds of ADL $(p=.002)$ and IADL $(p<.001)$ disability. We repeated these analyses excluding participants with stroke, heart failure, and cancer at baseline, and while associations remained, they were attenuated especially for the ADL disability outcome (data not shown).

The odds of worsening ADL disability were lowered by $19 \%$ per 1-point increase of baseline CVH score, while this for IADL disability was $16 \%$ lower over a 9-year follow-up among all participants (Table 3). Similar protective associations were also seen for participants who were free of disability at baseline.

Among participants without disability at baseline (Table 4), 1-point increase in the $\mathrm{CVH}$ score was associated with $14 \%$ lower hazard of ADL disability $(p=.005)$ and $9 \%$ lower hazard of IADL disability $(p=.007)$. Protective associations were observed for CVH modeled as tertiles, while associations with IADL were weaker.

In the adjusted longitudinal analysis between health behaviors and factors and disability outcomes, health behaviors were found to be associated with 29\% lower odds of ADL ( $p<$. $001)$ and $28 \%$ lower odds of IADL $(p<.001)$ disability, as well as decreased risk of worsening ADL $(p<.001)$ and IADL $(p<.001)$ disability (Supplementary Tables 2 and 3$)$. No significant associations were seen between health factor scores and disability.

\section{Discussion}

In this cohort of older men and women, better baseline CVH scores, assessed using four health behaviors (smoking status, physical activity, BMI, and diet quality) and three health factors (blood pressure, total cholesterol, and fasting plasma glucose), were associated with lower risk of ADL and IADL disability over a 9-year follow-up. In addition, participants with better CVH also had slower worsening of ADL and IADL disability. CVH was examined both as a continuous and categorical factor, and the results indicate that the reduced risk of disability was linear with incremental protection observed with higher scores of $\mathrm{CVH}$. 
Consistent with prior studies (Devulapalli et al., 2016; Dhamoon et al., 2015; García-

Hermoso et al., 2017), the current study confirms that higher CVH score is incrementally associated with lower risk of ADL disability over the 9-year follow-up period. Higher CVH was also inversely associated with the risk of IADL disability which may identify incipient declines in physical and cognitive functioning, and this study is the first to show that this protection is also observed longitudinally in older population. Furthermore, this is the first longitudinal study to show that $\mathrm{CVH}$, based on AHA guidelines, is a valid and powerful predictor of disability in non-American population. This would suggest that CVH index is a useful tool to assess health worldwide.

This study also showed slightly attenuated associations for participants with no disability at baseline compared with all participants, indicating potential reverse causality, so disabled participants at baseline tend to have a more sedentary lifestyle, and the physical activity of the component of the metric may be associated with disability. While this may be true, disability is a complex phenomenon and involves interactions between the biology orimpairments in body functions and structures and contextual factors such as the environment leading to restriction in participation in activities eventually resulting in disability (Schneidert, Hurst, Miller, \& Ustun, 2003).

Few investigations have focused on CVH in older populations. While AHA LS7 metric has been shown to be associated with chronic diseases and mortality, the effect could be modified by age. Yang and colleagues (2012) showed a significant interaction by age (<60 vs. 760 years) on associations between CVH and CVD mortality with greater protection among the younger age group, but the absolute numbers of preventable CVD deaths were higher in older participants. Using nationally representative data from the NHANES, Benjamin et al. (2017) reported that younger adults were more likely to meet a greater number of the ideal metrics than older adults and, except for the diet quality component, had a higher prevalence of ideal levels of CVH components. Our study population consisted of individuals above the age of 65 years and older present with a higher prevalence of disability; therefore, it is important to study whether better $\mathrm{CVH}$ at an older age is associated disability status. Our results show that higher CVH scores, even at an older age, have protective influences on reducing the risk of disability. Associations were attenuated after excluding participants with stroke, heart failure, and cancer at baseline, especially for the association between ADL disability and $\mathrm{CVH}$, suggesting that these disease conditions may be in the causal pathway and directly lead to higher risk of disability. Protective associations between $\mathrm{CVH}$ and worsening of disability status were also observed in the InCHIANTI cohort.

Strengths of this study include the prospective cohort setting which allowed us to examine the associations between baseline $\mathrm{CVH}$ on disability over 9 years. The use of two validated and well-documented measures of self-reported functional status, ADL which measures the basic skills needed for everyday living and IADL which examines skills required for independent living, is also a significant strength. In addition, the ability to examine potential confounders and covariates including chronic disease conditions and cognitive status is also a positive. Our study has some limitations, including a relatively small analytical sample size. Approximately $13 \%$ of participants who were eligible for these analyses were missing 
at least one of the CVH component. At baseline, these individuals were generally older, presented with poor measures of physical function, were more likely to be cognitive impaired and present with depression, and had a higher risk of mortality during follow-up years (Supplementary Table 4), which limits the generalizability of our findings. Almost $35 \%$ of participants were missing ADL and IADL disability information at 9-year follow-up, mostly due to death. Similar to those participants missing CVH information at baseline, these individuals were older, more likely to have poor physical function, be cognitive impairedand depressive, and additionally have poor health status for $\mathrm{CVH}$ metrics (Supplementary Table 5), which may have led to an underestimation of the association between $\mathrm{CVH}$ and disability.

In conclusion, we found an inverse association between higher $\mathrm{CVH}$ scores, as measured by the AHA LS7 metric, and disability in older individuals. Better CVH at baseline was also associated with lower odds of disability worsening over the 9-year follow-up. We have previously reported that this CVH score is significantly associated with all-cause and CVD mortality as well as performance-based measures of physical function. Here, we show that $\mathrm{CVH}$ is also strongly predictive of development of disability. This underscores the importance of targeting these health and behavior risk factors, particularly in the aged, to reduce morbidity and mortality. Our results need to be confirmed by other longitudinal investigations, and clinicians need to investigate future interventions targeted at improving $\mathrm{CVH}$ in older individuals with an overall goal of preventing disability and improving the quality of life.

\section{Supplementary Material}

Refer to Web version on PubMed Central for supplementary material.

\section{Acknowledgments}

Funding

The authors disclosed receipt of the following financial support for the research, authorship, and/or publication of this article: This study was supported by National Institute on Aging, grant No. R03AG048377. The InCHIANTI Study was supported as a "targeted project" (ICS 110.1/RF97.71) by the Italian Ministry of Health and in part by the U.S. National Institute on Aging (Contracts: 263 MD 9164 13, 263 MD 821336, N.1-AG-1-1, N.1-AG-1-2111, N01-AG-5-0002). None of the sponsoring institutions interfered with the collection, analysis, presentation, or interpretation of the data reported.

\section{References}

Bartali B, Turrini A, Salvini S, Lauretani F, Russo CR, Corsi AM, ... Guralnik JM (2004). Dietary intake estimated using different methods in two Italian older populations. Archives of Gerontology and Geriatrics, 38, 51-60. [PubMed: 14599704]

Benjamin EJ, Blaha MJ, Chiuve SE, Cushman M, Das SR, Deo R, ...Stroke Statistics S (2017). Heart disease and stroke statistics-2017 update: A report from the American Heart Association. Circulation, 135(10), e146-e603. doi:10.1161/CIR.0000000000000485 [PubMed: 28122885]

Crimmins EM (2004). Trends in the health of the elderly. Annual Review of Public Health, 25, 79-98.

Devulapalli S, Shoirah H, \& Dhamoon MS (2016). Ideal cardiovascular health metrics are associated with disability independently of vascular conditions. PLoS ONE, 11(2), e0150282. [PubMed: 26926808] 
Dhamoon MS, Dong C, Elkind MS, \& Sacco RL (2015). Ideal cardiovascular health predicts functional status independently of vascular events: The northern Manhattan study. Journal of the American Heart Association, 4(2), e001322. [PubMed: 25677566]

Dick J, Guiloff R, Stewart A, Blackstock J, Bielawska C, Paul E, \& Marsden C (1984). Mini-mental state examination in neurological patients. Journal of Neurology, Neurosurgery \& Psychiatry, 47, 496-499.

Ferrucci L, Bandinelli S, Benvenuti E, Iorio A, Macchi C, Harris TB, \& Guralnik JM (2000). Subsystems contributing to the decline in ability to walk: Bridging the gap between epidemiology and geriatric practice in the InCHIANTI study. Journal of the American Geriatrics Society, 48, 1618-1625. [PubMed: 11129752]

Folsom AR, Yatsuya H, Nettleton JA, Lutsey PL, Cushman M, \& Rosamond WD (2011). Community prevalence of ideal cardiovascular health, by the American heart association definition, and relationship with cardiovascular disease incidence. Journal of the American College of Cardiology, 57, 1690-1696. [PubMed: 21492767]

García-Hermoso A, Ramírez-Vélez R, Ramirez-Campillo R, \& Izquierdo M (2017). Relationship between ideal cardiovascular health and disability in older adults: The Chilean National Health Survey (2009-10). Journal of the American Geriatrics Society, 65, 2727-2732. [PubMed: 29067687]

Guralnik JM, Fried LP, Simonsick EM, Kasper JD, \& Lafferty ME (1995, 7). The women's health and aging study: Health and social characteristics of older women with disability (NIH Publication No. 95-4009). Bethesda, MD: National Institute on Aging.

Jin Y, Tanaka T, Bandinelli S, Ferrucci L, \& Talegawkar SA (2016). Overall cardiovascular health is associated with all-cause and cardiovascular disease mortality among older community-dwelling men and women. Journal of Aging and Health, 29, 437-453. doi:10.1177/0898264316635590 [PubMed: 27036884]

Jin Y, Tanaka T, Ma Y, Bandinelli S, Ferrucci L, \& Talegawkar SA (2017). Cardiovascular health is associated with physical function among older community dwelling men and women. The Journals of Gerontology. Series A, Biological Sciences and Medical Sciences, 72, 1710-1716.

Katz S, Ford AB, Moskowitz RW, Jackson BA, \& Jaffe MW (1963). Studies of illness in the aged: The index of ADL: A standardized measure of biological and psychosocial function. Journal of the American Medical Association, 185, 914-919. [PubMed: 14044222]

Lawton M, \& Brody EM (1969). Assessment of older people: Self-maintaining and instrumental activities of daily living. Gerontologist, 9, 179-186. [PubMed: 5349366]

Lloyd-Jones D, Hong Y, Labarthe D, Mozaffarian D, Appel L, Van Horn L, ... American Heart Association Strategic Planning Task Force and Statistics Committee. (2010). Defining and setting national goals for cardiovascular health promotion and disease reduction: The American heart association's strategic impact goal through 2020 and beyond. Circulation, 121, 586-613. [PubMed: 20089546]

Milaneschi Y, Bandinelli S, Corsi AM, Lauretani F, Paolisso G, Dominguez LJ, ... Talegawkar SA (2011). Mediterranean diet and mobility decline in older persons. Experimental Gerontology, 46, 303-308. [PubMed: 21111801]

Millán-Calenti JC, Tubío J, Pita-Fernández S, González-Abraldes I, Lorenzo T, Fernández-Arruty T, \& Maseda A (2010). Prevalence of functional disability in activities of daily living (ADL), instrumental activities of daily living (IADL) and associated factors, as predictors of morbidity and mortality. Archives of Gerontology and Geriatrics, 50, 306-310. [PubMed: 19520442]

Pisani P, Faggiano F, Krogh V, Palli D, Vineis P, \& Berrino F (1997). Relative validity and reproducibility of a food frequency dietary questionnaire for use in the Italian EPIC centres. International Journal of Epidemiology, 26(Suppl. 1), S152-160. [PubMed: 9126543]

Rebholz CM, Anderson CA, Grams ME, Bazzano LA, Crews DC, Chang AR, ... Appel LJ (2016). Relationship of the American Heart Association's impact goals (Life's Simple 7) with risk of chronic kidney disease: Results from the atherosclerosis risk in communities (ARIC) cohort study. Journal of the American Heart Association, 5(4), e003192. [PubMed: 27053058]

Schneidert M, Hurst R, Miller J, \& Ustun B (2003). The role of environment in the international classification of functioning, disability and health (ICF). Disability and Rehabilitation, 25, 588595. doi:10.1080/0963828031000137090 [PubMed: 12959332] 
StataCorp (2013). Stata Statistical Software: Release 13 College Station, TX:StataCorp LP.

Talegawkar SA, Jin Y, Kandula NR, \& Kanaya AM (2017). Cardiovascular health metrics among South Asian adults in the United States: Prevalence and associations with subclinical atherosclerosis. Preventive Medicine, 96, 79-84. [PubMed: 28007496]

Thacker EL, Gillett SR, Wadley VG, Unverzagt FW, Judd SE, McClure LA, ... Cushman M. (2014). The American Heart Association Life's Simple 7 and incident cognitive impairment: The reasons for geographic and racial differences in stroke (regards) study. Journal of the American Heart Association, 3(3), e000635. [PubMed: 24919926]

Trichopoulou A, Costacou T, Bamia C, \& Trichopoulos D (2003). Adherence to a Mediterranean diet and survival in a Greek population. The New England Journal of Medicine, 348, 2599-2608. [PubMed: 12826634]

United Nations, Department of Economic and Social Affairs, Population Division. (2015). World population ageing (ST/ESA/SER.A/390). New York: United Nations.

U.S. Census Bureau. (2015). Millennials outnumber baby boomers and are far more diverse, census bureau reports Retrieved from https://www.census.gov/news-room/press-releases/2015/ cb15-113.html

Vincent GK, \& Velkoff VA (2010). The next four decades: The older population in the United States: 2010 to 2050 Washington, DC: U.S. Department of Commerce, Economics and Statistics Administration, U.S. Census Bureau.

Yang Q, Cogswell ME, Flanders WD, Hong Y, Zhang Z, Loustalot F, ... Hu FB (2012). Trends in cardiovascular health metrics and associations with all-cause and CVD mortality among us adults. Journal of the American Medical Association, 307, 1273-1283. doi:10.1001/jama.2012.339 [PubMed: 22427615] 
Table 1.

Baseline Sociodemographic and Health Characteristics by the Tertiles of Overall Cardiovascular Health Score Among InCHIANTI Participants Aged 65 Years and Older.

\begin{tabular}{|c|c|c|c|c|c|}
\hline & \multicolumn{4}{|c|}{ Overall cardiovascular health score } & \multirow[b]{3}{*}{$p$ value } \\
\hline & Total & First tertile & Second tertile & Third tertile & \\
\hline & Score:0-12 & Score: $0-6$ & Score: $7-8$ & Score: 9-12 & \\
\hline$n$ & 925 & 250 & 375 & 300 & \\
\hline \multicolumn{6}{|l|}{ Covariates } \\
\hline Female $(\%)$ & 55 & 56 & 58 & 51 & .177 \\
\hline Age (years) & $74.1 \pm 6.7$ & $74.7 \pm 6.8$ & $74.2 \pm 6.5$ & $73.4 \pm 6.6$ & .054 \\
\hline Education (years) & $5.5 \pm 3.2$ & $5.5 \pm 3.3$ & $5.5 \pm 3.1$ & $5.6 \pm 3.4$ & .919 \\
\hline Impaired cognition $^{a}(\%)$ & 24 & 25 & 24 & 22 & .603 \\
\hline Depressed $\operatorname{mood}^{b}, n=917(\%)$ & 30 & 33 & 32 & 27 & .177 \\
\hline Number of chronic diseases $(\%)$ & & & & & $<.001$ \\
\hline 0 & 14 & 8 & 11 & 22 & \\
\hline 1 & 35 & 24 & 39 & 38 & \\
\hline 2 & 31 & 31 & 32 & 28 & \\
\hline 23 & 21 & 37 & 18 & 12 & \\
\hline \multicolumn{6}{|l|}{ Outcome } \\
\hline At least one ADL disability (\%) & 4 & 5 & 5 & 1 & .016 \\
\hline At least one IADL disability (\%) & 19 & 27 & 21 & 11 & $<.001$ \\
\hline \multicolumn{6}{|l|}{ Components of cardiovascular health } \\
\hline Current smoker (\%) & 14 & 25 & 14 & 6 & $<.001$ \\
\hline Physical activity (\%) & & & & & $<.001$ \\
\hline Inactive & 17 & 35 & 15 & 3 & \\
\hline Light active & 44 & 50 & 51 & 30 & \\
\hline Active & 39 & 14 & 34 & 67 & \\
\hline Body mass index $\left(\mathrm{kg} / \mathrm{m}^{2}\right)$ & $27.5 \pm 4.0$ & $29.6 \pm 4.3$ & $27.6 \pm 3.9$ & $25.8 \pm 3.1$ & $<.001$ \\
\hline Mediterranean diet score & $4.5 \pm 1.6$ & $3.7 \pm 1.6$ & $4.4 \pm 1.6$ & $5.2 \pm 1.4$ & $<.001$ \\
\hline Total cholesterol (mg/dL) & $219 \pm 38.8$ & $235 \pm 39.0$ & $222 \pm 35.0$ & $203 \pm 37.0$ & $<.001$ \\
\hline Systolic blood pressure $(\mathrm{mm} \mathrm{Hg})$ & $151 \pm 19.5$ & $155 \pm 16.9$ & $152 \pm 19.0$ & $145 \pm 20.8$ & $<.001$ \\
\hline Diastolic blood pressure (mm Hg) & $84.1 \pm 8.5$ & $85.6 \pm 7.7$ & $84.7 \pm 8.4$ & $82.3 \pm 9.0$ & $<.001$ \\
\hline Fasting plasma glucose $(\mathrm{mm} / \mathrm{dL})$ & $89(82-100)$ & $101(87-121)$ & $88(81-97)$ & $86(80-93)$ & $<.001$ \\
\hline
\end{tabular}

Note. Data are reported as mean \pm standard deviation, percentage, and median (interquartile range). ADL = activities of daily living; IADL $=$ instrumental activities of daily living. The overall cardiovascular health score ranges 0-12 in this cohort.

Impaired cognition defined as Mini-Mental State Examination score $<24$.

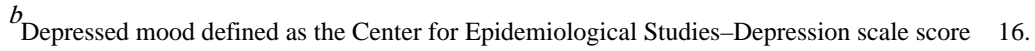


Table 2.

Odds Ratio and 95\% Confidence Intervals of ADL and IADL Disability Comparing Across the Tertiles of Baseline Overall CVH Score Among InCHIANTI Participants Aged 65 Years and Older Over a 9-Year Follow-Up Using GEE Model.

\begin{tabular}{|c|c|c|c|c|c|c|c|c|}
\hline \multirow[b]{2}{*}{ Overall CVH score } & \multicolumn{4}{|c|}{ ADL } & \multicolumn{4}{|c|}{ IADL } \\
\hline & Cases $^{a}$ & $n^{a}$ & OR $(95 \% \mathrm{CI})$ & $p$ value & Cases $^{a}$ & $n^{a}$ & OR $(95 \% \mathrm{CI})$ & $\begin{array}{c}p \\
\text { value }\end{array}$ \\
\hline All participants $b$ & 183 & 925 & & & 501 & 925 & & \\
\hline Per 1 point increase & & & $0.77[0.69,0.86]$ & $<.001$ & & & $0.83[0.77,0.89]$ & $<.001$ \\
\hline \multicolumn{9}{|l|}{ Tertile score } \\
\hline $0-6$ & 73 & 250 & 1.00 & & 163 & 250 & 1.00 & \\
\hline $7-8$ & 75 & 375 & $0.60[0.39,0.92]$ & .019 & 201 & 375 & $0.62[0.46,0.84]$ & .002 \\
\hline $9-12$ & 35 & 300 & $0.33[0.20,0.54]$ & $<.001$ & 137 & 300 & $0.47[0.34,0.66]$ & $<.001$ \\
\hline \multicolumn{9}{|c|}{ Participants free of disability at baseline ${ }^{c}$} \\
\hline Per 1 point increase & 146 & 888 & $0.83[0.73,0.93]$ & .002 & 322 & 746 & $0.87[0.81,0.94]$ & $<.001$ \\
\hline \multicolumn{9}{|l|}{ Tertile score } \\
\hline $0-6$ & 60 & 237 & 1.00 & & 95 & 182 & 1.00 & \\
\hline $7-8$ & 55 & 355 & $0.51[0.32,0.82]$ & .005 & 123 & 297 & $0.64[0.46,0.89]$ & .009 \\
\hline $9-12$ & 31 & 296 & $0.38[0.23,0.64]$ & $<.001$ & 104 & 267 & $0.58[0.42,0.82]$ & .002 \\
\hline
\end{tabular}

Note. Model adjusted for age, sex, years of education (for IADL only), impaired cognition, depressed mood, and number of chronic disease. GEE = generalized estimating equations; $\mathrm{CVH}=$ cardiovascular health; $\mathrm{ADL}=$ activities of daily living; IADL = instrumental activities of daily living; OR $=$ odds ratio $; \mathrm{CI}=$ confidence intervals

${ }_{n}^{a}=$ baseline sample size, cases $=$ number of disability over a 9-year follow-up.

${ }^{b}$ Baseline $n=917$ for ADL and IADL analysis due to eight missing on depressed mood.

$c$ Baseline $n=885$ for ADL analysis due to three missing on depressed mood; baseline $n=745$ for IADL analysis due to one missing on depressed mood. 
Table 3.

Odds Ratio and 95\% Confidence Intervals of Worsening ADL and IADL Disability Per 1 Unit Baseline Overall CVH Score Increase Among InCHIANTI Participants Aged 65 Years and Older Over a 9-Year Follow-Up Using GEE Model.

\begin{tabular}{|c|c|c|c|c|}
\hline \multirow[b]{2}{*}{ Overall CVH score } & \multicolumn{2}{|c|}{ Worsening ADL disability } & \multicolumn{2}{|c|}{ Worsening IADL disability } \\
\hline & OR $(95 \%$ CI $)$ & $p$ value & OR $(95 \% \mathrm{CI})$ & $p$ value \\
\hline \multicolumn{5}{|l|}{ All participants } \\
\hline Per 1 point increase & $0.81[0.73,0.91]$ & $<.001$ & $0.84[0.78,0.90]$ & $<.001$ \\
\hline \multicolumn{5}{|l|}{ Tertile score } \\
\hline $0-6$ & 1.00 & & 1.00 & \\
\hline $7-8$ & $0.56[0.36,0.88]$ & .012 & $0.50[0.36,0.70]$ & $<.001$ \\
\hline $9-12$ & $0.37[0.22,0.63]$ & $<.001$ & $0.48[0.34,0.67]$ & $<.001$ \\
\hline \multicolumn{5}{|c|}{ Participants free of disability at baseline } \\
\hline Per 1 point increase & $0.83[0.73,0.93]$ & .002 & $0.86[0.79,0.94]$ & .001 \\
\hline \multicolumn{5}{|l|}{ Tertile score } \\
\hline $0-6$ & 1.00 & & 1.00 & \\
\hline $7-8$ & $0.51[0.32,0.83]$ & .007 & $0.59[0.41,0.85]$ & .005 \\
\hline $9-12$ & $0.38[0.22,0.64]$ & $<.001$ & $0.55[0.38,0.80]$ & .002 \\
\hline
\end{tabular}

Note. Model adjusted for age (years), sex, years of education (for IADL only), impaired cognition, depressed mood, and number of chronic disease. $\mathrm{GEE}=$ generalized estimating equations; $\mathrm{CVH}=$ cardiovascular health; $\mathrm{ADL}=$ activities of daily living; $\mathrm{IADL}=$ instrumental activities of daily living; $\mathrm{OR}=$ odds ratio; $\mathrm{CI}=$ confidence intervals. 
Table 4.

Hazard Ratio and 95\% Confidence Intervals of ADL and IADL Disability Across the Tertiles of Baseline Overall CVH Score Among InCHIANTI Participants Aged 65 Years and Older Over a 9-Year Follow-Up Free of ADL or IADL Disability at Baseline.

\begin{tabular}{|c|c|c|c|c|c|c|c|c|}
\hline \multirow{2}{*}{$\begin{array}{l}\text { Overall CVH } \\
\text { score }\end{array}$} & \multicolumn{4}{|c|}{ ADL disability $(n=822)$} & \multicolumn{4}{|c|}{ IADL disability $(n=700)$} \\
\hline & Cases & $n$ & HR $(95 \% \mathrm{CI})$ & $p$ value & Cases & $n$ & HR $(95 \% \mathrm{CI})$ & $p$ value \\
\hline $\begin{array}{l}\text { Per } 1 \text { point } \\
\text { increase }\end{array}$ & & & $0.86[0.77,0.95]$ & 0.005 & & & $0.91[0.86,0.98]$ & .007 \\
\hline \multicolumn{9}{|l|}{ Tertile score } \\
\hline $0-6$ & 59 & 222 & 1.00 & & 95 & 172 & 1.00 & \\
\hline $7-8$ & 55 & 327 & $0.50[0.33,0.75]$ & 0.001 & 123 & 279 & $0.74[0.55,0.98]$ & .035 \\
\hline $9-12$ & 31 & 273 & $0.47[0.30,0.73]$ & 0.001 & 104 & 249 & $0.74[0.55,0.98]$ & .038 \\
\hline
\end{tabular}

Note. Model adjusting for age, sex, years of education (for IADL only), impaired cognition, depressed mood, and number of chronic diseases. GEE = generalized estimating equations; $\mathrm{CVH}=$ cardiovascular health; $\mathrm{ADL}=$ activities of daily living; IADL = instrumental activities of daily living; $\mathrm{HR}=$ hazard ratio $\mathrm{CI}=$ confidence intervals . 